\title{
Childhood Cardiomyopathies: A Study in Tertiary Care Hospital in Upper Egypt
}

\author{
Mohamed Abd Elaal Bakeet ${ }^{1}$, Montaser Mohamed Mohamed ${ }^{2}$, Ahmed Ahmed Allam³ ${ }^{3}$ Rania Gamal ${ }^{4}$
}

${ }^{1}$ M.D., Professor of Pediatrics, Faculty of Medicine, Sohag University, Egypt

${ }^{2}$ M.D., Lecturer of Pediatrics, Faculty of Medicine, Sohag University, Egypt

${ }^{3}$ M.D., Lecturer of Clinical Pathology, Faculty of Medicine, Sohag University, Egypt

${ }^{4}$ M.D., Assistant Lecturer of Pediatrics, Faculty of Medicine, Sohag University, Egypt

\section{Type of article: Original}

\begin{abstract}
Introduction: Cardiomyopathy (CMP) is defined by the World Health Organization (WHO) as a disease of the myocardium associated with cardiac dysfunction. An understanding of CMP is very important, as it is a common cause of heart failure in children, and the most common indication for heart transplantation in children older than one year, but data on CMP in Egypt are scarce. The aim of this study was to determine the number, risk factors, clinical presentation, complications and outcome of different types of childhood cardiomyopathies in Sohag University Hospital.

Methods: This cross-sectional hospital based study enrolled fifty children with Cardiomyopathy in Pediatric Departments, Neonatal Care Units, and Cardiac Outpatient Clinics in Sohag University Hospital from March 01, 2014 to February 28, 2015.

Results: Cases with Dilated Cardiomyopathy (DCMP) were 38 (76\%), and those who had Hypertrophic Cardiomyopathy (HCMP) were 12 (24\%). Dyspnea was the most common presenting complaint in $71 \%$ of cases. In cases with DCMP, the mean EF was 33.8, and FS was 17.11, while in cases with HCMP, the mean EF was 70.75 , FS was 37 . Fifty percent of cases were found to have moderate to severe PHT. Serum CK-MB was elevated in $3(6 \%)$ cases, while serum Troponin I was elevated in $2(4.2 \%)$ cases who diagnosed as having myocarditis. Viral myocarditis was the most common identified etiological agent responsible for $14(37 \%)$ cases with DCMP.

Conclusions: CMP represents a considerable percentage of children with cardiac disorders. DCMP is the most common type, usually presented with congestive heart failure, and the most common cause is myocarditis. LCarnitine profile was normal in all cases, despite its routine use. Pediatricians need to raise their clinical suspicion to CMPs, as atypical presentations are not uncommon. To do screening for other family members, cardiac enzymes (CK-MB, Troponin I) have to be done in all newly diagnosed CMP cases, along with a revision of the routine prescription of L-Carnitine.
\end{abstract}

Keywords: Childhood cardiomyopathy, Dilated cardiomyopathy, Hypertrophic Cardiomyopathy, Egypt

\section{Introduction}

Cardiomyopathy (CMP) is defined by the World Health Organization (WHO) as a disease of the myocardium associated with cardiac dysfunction (1). An understanding of CMP is very important, as it is a common cause of heart failure in children and the most common indication for heart transplantation in children older than 1 year (2). The WHO classifies CMP as dilated (DCMP), hypertrophic (HCMP), restrictive (RCMP), and arrhythmogenic right ventricular (ARVC), or unclassified (1). The most common is DCMP (58\%), followed by HCMP (30\%). There were relatively few cases of RCMP $(5 \%)$ and $\operatorname{ARVC}(5 \%)(3,4)$. There are multiple factors that may be implicated in the aetiology of CMP such as: familial or genetic causes, as many children with HCMP (60\%) and DCMP (48\%) have a family history of the disease $(5,6)$. Also it may be due to acquired causes such as inflammatory or infectious

\section{Corresponding author:}

Dr. Montaser Mohamed Mohamed, Faculty of Medicine, Sohag University, Egypt.

Tel.: +20.1144117895, Email: montaser_dabah@yahoo.com

Received: July 26, 2016, Accepted: October 27, 2016, Published: November 2016

iThenticate screening: October 27, 2016, English editing: November 06, 2016, Quality control: November 10, 2016

(C) 2016 The Authors. This is an open access article under the terms of the Creative Commons Attribution-NonCommercialNoDerivs License, which permits use and distribution in any medium, provided the original work is properly cited, the use is non-commercial and no modifications or adaptations are made. 
diseases, autoimmune diseases, and toxin reactions. Moreover, there are other important causes as neuromuscular disorders, and metabolic diseases $(7,8)$. Idiopathic CMP is called so, when the aetiology is unknown (3). The symptoms of CMP may differ in every child according to age, type, and severity (3). In DCMP, Infants and children generally present with signs of congestive heart failure such as tachypnea, labored breathing, poor appetite, and slow weight gain. In older children, there may be signs of poor exercise tolerance and gastrointestinal distress (7). While HCMP has a wide range of clinical presentations, from few or even no symptoms to serious complications, including syncope, heart failure and sudden death (3), heart murmurs are not always present in children with CMP (3). Because CMP is not easily detected on physical examination, it is often diagnosed in its later stages (9). Thus, it is recommended that all first degree relatives should be screened for CMP, as early detection can affect treatment modality, and long term prognosis (3). Diagnosis of CMP is usually reached through noninvasive procedures such as, chest radiography which reveals cardiomegaly, and increased pulmonary vascular markings (7). Electrocardiography (ECG) may reveal various types of arrhythmias. Echocardiography is the most informative noninvasive test for diagnosing and classifying CMP, and determining the degree of dysfunction in the heart muscle (3). Genetic studies may be needed especially in familial CMP, and certain cardiac biomarkers may be needed to determine the severity in cardiac dysfunction. Invasive procedures such as cardiac catheterization are sometimes necessary to determine heart function, and to obtain endomyocardial sampling especially in children who are candidate for heart transplantation (10). The overall long-term prognosis of pediatric patients depends on the CMP type, and disease stage (3). The 9-year survival rate was estimated to be $69.8 \%$ in DCMP, $90.3 \%$ in HCMP, $47.2 \%$ in RCMP, and $42.0 \%$ in other CMP patients (12). The objectives of the present study were to determine the number, risk factors, clinical presentation, complications and outcome of different types of childhood cardiomyopathies in Sohag University Hospital.

\section{Material and Methods}

\subsection{Study Design}

This was a cross-sectional hospital based study that was conducted in the Pediatric Department, Neonatal Care Unit and Cardiac Outpatient Clinic in Sohag University Hospital. The study period was one year which extended from 13-2014 to 28-2- 2015 .

\subsection{Selection Criteria}

Inclusion criteria were children under 12 years old who were diagnosed as having definite CMP, while exclusion criteria included children with congenital heart diseases, rheumatic heart diseases or other known cardiac diseases.

\subsection{Data collection}

Data included complete medical history of the patients with special attention to cardiac symptoms particularly those which are in favor for diagnosis of CMP, such as symptoms of heart failure (dyspnea, palpitation, exercise intolerance, interrupted feeding, failure to thrive and recurrent chest infections) and syncope. Data about risk factors of CMP, such as viral upper respiratory tract infections, chemotherapeutic agents, neuromuscular diseases and metabolic diseases are also included in addition to family history of CMP. After gathering the previous data, the patients were subjected to general and cardiac examination. Results of investigating cardiac enzymes (CPK-MB and troponin) and serum L-Carnitine level were fulfilled. In addition to ECG and chest $\mathrm{x}$ - ray results. The diagnosis of DCMP by echocardiography was based on the presence of left ventricular dilatation and reduced systolic function with an ejection fraction $<50 \%$ or, more stringently, $<45 \%$, with or without mitral regurgitation. Also Left ventricular (LV) wall thickness $>2$ standard deviations above the body surface area-corrected mean (z score) was a base for the diagnosis. Pericardial effusion, especially in myocarditis and heart rhythm irregularities was a base to make the diagnosis. In addition, assessing the pattern of hypertrophy (usually asymmetric preferentially affecting the anterior interventricular septum), global systolic function, diastolic performance, presence and mechanism of outflow tract obstruction, atrial dimensions, and valvular function (8) was done.

\subsection{Statistical analysis}

Both clinical, laboratory and radiological data were analyzed using STATA intercooled version 12.1. Quantitative data were analyzed, using student t-test to compare means of two groups. When the data was not normally distributed Mann-Whitney test was used. Qualitative data were compared using Chi square test. P value was considered significant if it was less than 0.05 . 


\subsection{Ethical consideration}

Approval of Sohag Faculty of Medicine research ethics committee has been obtained, and verbal consent from guardians of patients were also obtained.

\section{Results}

\subsection{General findings}

During the period of the study, there were 50 children who were diagnosed as having cardiomyopathy presented to our outpatient's clinic, inpatient paediatric department and neonatal care unit. The mean age of the studied children was 3.5 years with age range from 5 days to 12 years. Concerning the sex distribution of the studied children, there was no significant difference, 27 (46\%) children were males and $23(54 \%)$ were females. Consanguinity between parents of studied children was present in $32(64 \%)$ patients, while family history of similar disease was present in $11(22 \%)$ patients.

\subsection{Types of cardiomyopathies in the studied patients}

During the period of study, 50 cases were diagnosed as having CMP. The numbers of patients who have dilated cardiomyopathy (DCMP) were 38 (76\%), and those who have hypertrophic cardiomyopathy (HCMP) were 12 (24\%). Table 1 shows that dyspnea is the most common presenting complaint in DCMP (71\%) while poor or interrupted feeding was the most presenting complaint in the HCMP (75\%). Head and neck examination was normal in $46(92 \%)$ patients, while dysmorphic features were noted in $4(8 \%)$ cases. From Table 2 we found that systolic murmur could be heard in 24 (48\%) patients; 22 of them have DCMP, while no murmur could be heard in 18 (36\%), nine patients have DCMP and nine patients have HCMP. The most common finding in chest $\mathrm{x}$-ray was increased cardiothoracic ratio with increased lung vasculature $(60 \%)$ while pneumonia was found in $6(12 \%)$ patients. Twentytwo percent of patients had normal chest $\mathrm{x}$-ray. Increased cardiothoracic ratio and increased lung vasculature and pneumonia were seen in $4 \%$ of patients while $2 \%$ of them showed increased cardiothoracic ratio and increased lung vasculature \& right pleural effusion in the chest x-ray. In ECG, sinus tachycardia was the most common ECG finding $(60 \%)$ followed by normal ECG (20\% of cases). The other ECG findings were left ventricular hypertrophy $(10 \%)$, arrhythmia (4\%), supraventricular tachycardia (4\%), and inverted T-wave and elevated ST segment (2\%). Figure 1 shows the ejection fraction of the studied children. As regards to echocardiographic findings, we found that the mean ejection fraction (EF) in patients with DCMP was 33.8 (with range from 20 to 40) in about 32 patients (84.21\%). Also, we found that the mean fractional shortening (FS) in patients with DCMP was 17.11 (with range from 9 to 26) in about 36 patients (94.7\%) with DCMP. On the other hand, the mean EF in patients with HCMP was about 70.75 with range from $60-77$, and the mean FS was 37.5 with range from 26- 43. Regarding pulmonary pressure, we found that 24 (48\%) patients had pulmonary pressure ranged from $15-30 \mathrm{mmHg}$ (mild PHT), 20 (40\%) patients had pulmonary pressure ranged from $30-40 \mathrm{mmHg}$ (moderate PHT), the remaining $5(10 \%)$ patients had pulmonary pressure more than $40 \mathrm{mmHg}$ (severe PHT), and only one (2\%) patient had pulmonary pressure less than $15 \mathrm{mmHg}$. Serum CK-MB was carried out in all cases, and it was elevated in $3(6 \%)$ cases, while serum Troponin I was carried out in $48(96 \%)$ cases, and it was elevated in $2(4.2 \%)$ cases who diagnosed as having myocarditis. LCarnitine Profile was carried out in 46 (92\%) cases with no detected abnormalities in any of them. We have detected the aetiology of CMP in 17 (34\%) patients. Suspected viral myocarditis was the most common identified etiological agent responsible for 14 (37\%) cases with DCMP, while 3 (25\%) cases with HCMP have identified causes; two cases are infants of diabetic mothers (IDM), and one case was diagnosed to have Friedreich's ataxia. CMP is a chronic illness, and nearly all (98\%) of our cases admitted at least once, $30(60 \%)$ cases twice at least, and $9(18 \%)$ cases more than two admissions. At the end of the period of our study, $5(10 \%)$ cases had died (1-year survival rate $90 \%)$. The main causes of death are intractable heart failure (3 cases), fatal arrhythmia (1 case), and brain infarction (1 case).

Table 1. Presenting complaints of studied children.

\begin{tabular}{|l|l|l|l|l|}
\hline Presenting complaints & Dilated CMP $(\mathrm{n}=38)$ & Hypertrophic CMP $(\mathrm{n}=12)$ & Total & $\mathrm{p}$-value \\
\hline Accidently discovered & $1(2.63 \%)$ & 0 & $1(2.00 \%)$ & 0.57 \\
\hline Dyspnea & $27(71.05 \%)$ & $3(25.00 \%)$ & $30(60.00 \%)$ & \\
\hline Poor or interrupted feeding & $3(7.89 \%)$ & $9(75.00 \%)$ & $12(24.00 \%)$ \\
\hline Cough and recurrent Chest infection & $3(7.89 \%)$ & 0 & $3(6.00 \%)$ \\
\hline Palpitation & $2(5.26 \%)$ & 0 & $2(4.00 \%)$ \\
\cline { 1 - 4 } Abdominal pain & $1(2.63 \%)$ & 0 & $1(2.00 \%)$ \\
\hline Chronic diarrhea & $1(2.63 \%)$ & 0 & $1(2.00 \%)$ & \\
\hline
\end{tabular}


Table 2. Cardiac examination of studied children.

\begin{tabular}{|l|l|l|l|l|}
\hline Examination & DCMP $(\mathrm{n}=38)$ & HCMP $(\mathrm{n}=12)$ & Total & p-value \\
\hline No murmur & $9(23.68 \%)$ & $9(75.00 \%)$ & $18(36.00 \%)$ & \multirow{2}{*}{0.03} \\
\cline { 1 - 5 } Pansystolic murmur & $22(57.89 \%)$ & $2(16.67 \%)$ & $24(48.00 \%)$ & \\
\hline Irregular heart sound & $3(7.89 \%)$ & 0 & $3(6.00 \%)$ & \\
\hline Muffled heart sound & $3(7.89 \%)$ & $1(8.33 \%)$ & $4(8.00 \%)$ & \\
\cline { 1 - 3 } Pansystolic murmur \& Muffled heart sound & $1(2.63 \%)$ & 0 & $1(2.00 \%)$ & \\
\hline
\end{tabular}

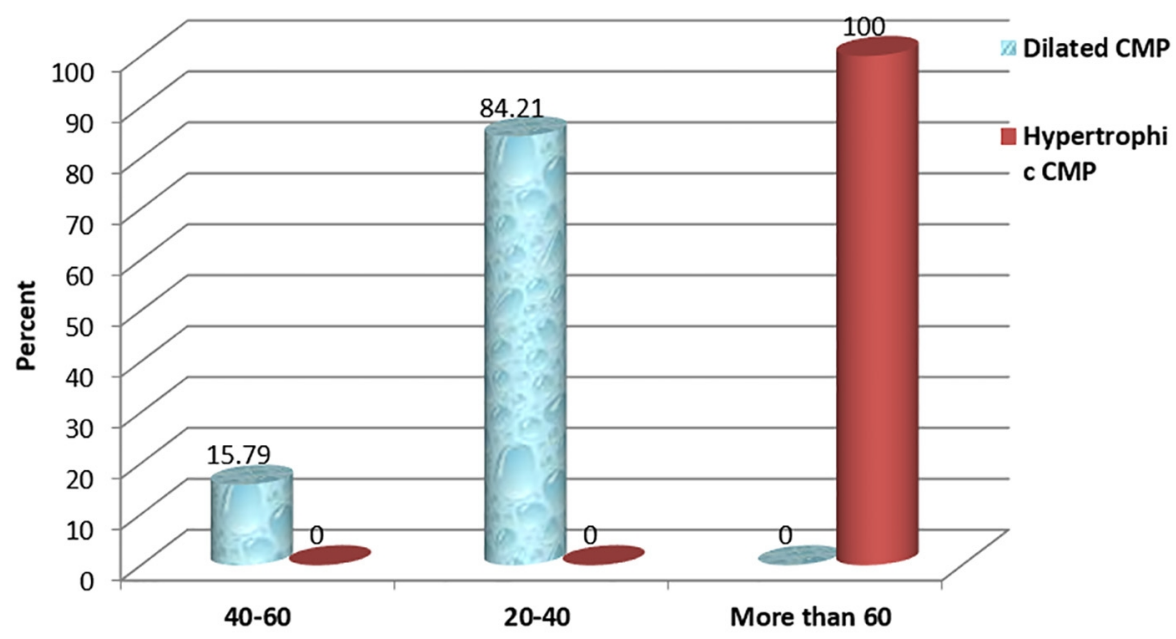

Figure 1. Ejection fraction of the studied children

\section{Discussion}

An understanding of CMP is very important, as it is a common cause of heart failure in children, and the most common indication for heart transplantation in children older than 1 year (2), but data on CMP in Egypt are scarce, as there is no national registry (5). The present study at Sohag University Hospital performed a clinical analysis, and reviewed the data of 50 children having CMP regarding the number, clinical presentation, possible etiological factors, complications and outcome of different types of childhood CMP. . Children with CMP represented $8 \%$ of all children with cardiac diseases who either admitted (during period of study) in the pediatric department, neonatal care unit, or those who followed up, in the cardiac outpatient clinic in Sohag University Hospital. This shows the importance of study of this disease, and the result is close to that of an El-Masry study (6.6\%) (5). Diagnosis of CMP is usually reached through noninvasive procedures. Echocardiography is the most informative noninvasive test for diagnosing and classifying CMP, and determining the degree of dysfunction in the heart muscle (3). The majority of the patients of our study (76\%) had dilated cardiomyopathies (DCMP), while the rest (24\%) had hypertrophic cardiomyopathies (HCMP). In Young`s study; the majority of the patients (66.4\%) also had DCMP, HCMP represented (23.5\%), RCMP (6.5\%), and others (3.6\%) (3). According to the United States (US) Pediatric Cardiomyopathy Registry, DCMP was the most common (58\%), followed by HCMP (30\%), RCMP (5\%) and ARVC (5\%) (4). In a study on Korean children, DCMP was (66.43\%), HCMP (23.47\%), RCMP (6.50\%), and others (3.61\%) (12). All these studies coincide with the results of our study in that, the most common type of CMP is DCMP followed by HCMP. None of the patients of our study had RCMP or ARVC, and this can be explained by the rarity of these disorders in children, and the small number of cases of our study. The mild differences between varied studies probably reflect selection bias rather than true frequencies. Concerning family history, consanguinity was present in $64 \%$ of patients. This result has limited importance as the Egyptian population is characterized by a high consanguinity (25-70\%) and a high percentage of first-cousin marriages (13). Family history of similar disease was present in $22 \%$ of our patients. Results are higher than that of El Masry's study (6.5\%) (5), and the Korean study $(8.3 \%)$, while are much lower than that of Towbin's study in which $50 \%$ to $60 \%$ of patients with HCMP and $30 \%$ to $48 \%$ of those with DCMP have a family history (14). The differences can be explained in that, many of the familial cases in Towbin's study were asymptomatic and diagnosed by screening and genetic studies. Such advanced methods are unfortunately unavailable in developing countries like Egypt. With advances in genetic study, the percentages of CMPs with a familial tendency are expected to increase in the future. In our study, the etiology of CMP could not be found in most cases. (66\%). Viral myocarditis was the most common identified etiological agent 
responsible for $37 \%$ of cases with DCMP, while $25 \%$ of cases with HCMP have identified causes (IDM, Friedreich`s ataxia). According to the United States Pediatric Cardiomyopathy Registry, the vast majority of CMP cases lack a known cause. In DCMP, only 34\% had a known cause, from which $16 \%$ had myocarditis, $9 \%$ with a neuromuscular disorder, $5 \%$ with familial cardiomyopathy, $4 \%$ with inborn errors of metabolism, and $1 \%$ with malformation syndrome. In HCMP, only $26 \%$ had a known cause: $9 \%$ with malformation syndrome, $9 \%$ with inborn errors of metabolism, and $8 \%$ with neuromuscular disorders (4). Our results agree with these studies in general, although due to the available advanced diagnostic modalities, it is expected that more etiological agents will be discovered in developed countries compared to Egypt. Concerning presentation, children with CMPs have a wide range of clinical presentation. The majority of cases with DCMP (71\%) presented with dyspnea compared to only $25 \%$ of HCMP cases. The results are close to that of the United States Pediatric Cardiomyopathy Registry (4). Some (10\%) of our cases had atypical presentations; $4 \%$ of them presented by palpitation, $2 \%$ with abdominal pain, $2 \%$ with chronic diarrhoea, while $2 \%$ had been accidentally discovered. This highlights the importance of studying CMP, as it should be kept in mind in such cases. Findings on cardiac examination are variable, with no detected abnormalities in $36 \%$ of cases, which signifies considering CMP even in cases of free cardiac examination, especially in high risk groups such as family members, where screening by echocardiography or genetic analysis must be performed. Echocardiography is the most informative noninvasive test for diagnosing and determining the degree of dysfunction in the heart muscle (3). The mean EF of cases with DCMP in our study was $33.8 \%$, and FS was $17 \%$, values which reflect the impaired cardiac function. Serum CK-MB was carried out in all cases, and it was elevated in $6 \%$ of cases, while serum Troponin I was carried out in $96 \%$ of cases, and was elevated in $4.2 \%$ of cases who were diagnosed as having myocarditis. It is advisable that levels of CK-MB and Troponin I be measured in the serum of all patients with CMP, as this may provide important clues to its aetiology (3). L-Carnitine Profile was carried out in $92 \%$ of cases with no detected abnormalities in any of them. L-Carnitine is traditionally prescribed to all cases of DCMP for the possibility of its deficiency, without carrying out a carnitine profile. Based on our study, such rationale must be revised. L-Carnitine supplementation was prescribed in $46 \%$ of the cases of our study, which is much larger than the results of Towbin's study in which only $15 \%$ of cases received it (14). L-Carnitine is traditionally prescribed to all cases of DCMP for the possibility of its deficiency, without carrying out a carnitine profile. In our study, we measured L-Carnitine profile in $92 \%$ of cases, in which no abnormalities were detected in any of them, making its use of questionable value. None of the cases of our study has undergone cardiac transplantation, ventricular assist device (VAD), intra-aortic balloon pump (IABP), or connected to ECMO. In a similar Korean study, 3\% of patients underwent cardiac transplantation, $1 \%$ had a VAD, and $0.3 \%$ had IABP (12). In an American study, approximately 5\% of patients underwent cardiac transplantation, 2\% IABP, 2\% ECMO, and $1 \%$ VAD (17). The numbers of patients treated with surgical or mechanical support have shown an increase in recent years, and these therapeutic trials along with experience might have an impact on the prognosis of CMP in the future. Egypt is a developing country with a low national outcome, and we lack these expensive, highly sophisticated interventions. CMP is a chronic illness, and nearly all of our cases admitted at least once, $42 \%$ twice and $18 \%$ more than two admissions. The results demonstrate the economic burden of such cases, and the load on the medical system, and encourage the research for new options in treatment. The mortality rate was $10 \%$ of cases at the end of our study period. Our results are close to that of Towbin's study where the 1 -year rate of death was $13 \%$ (14), and better than that of a Korean study (19.8\% at the end of the first year), however, results could not be compared to as long a term as the Korean study (death rate was $26.7 \%$ after eight years follow up) (12).

\section{Conclusions}

Despite limitations, this study is the first one which describes characterization of Pediatric CMP in Sohag Governorate, and one of few studies about CMP in Egypt. We found that CMP represents a considerable percentage of children with cardiac disorders. DCMP is the most common type, usually presented with congestive heart failure, but some cases have atypical presentations, and the most common cause is myocarditis. Echocardiography is the gold standard diagnostic method. L-Carnitine profile was normal in all cases, and its routine use needs to be revised. Most patients receive anti-failure treatment, and unlike other developed countries, surgical and mechanical replacement therapy are lacking. These findings are very important in the diagnosis, management and counseling of patients with CMPs.

\section{Acknowledgements:}

The authors gratefully thank administers of Sohag University Hospital and members of Pediatric Department for their support and providing technical and laboratory help in data collection. 


\section{Conflict of Interest:}

There is no conflict of interest to be declared.

\section{Authors' contributions:}

All authors contributed to this project and article equally. All authors read and approved the final manuscript.

\section{References:}

1) Richardson P, McKenna W, Bristow M, Maisch B, Mautner B, O'Connell J, et al. Report of the 1995 World Health Organization/ International Society and Federation of Cardiology Task Force on the definition and classification of cardiomyopathies. Circulation. 1996; 93(5): 841-2. doi: 10.1161/01.CIR.93.5.841. PMID: 8598070.

2) Wilkinson JD, Landy DC, Colan SD, Towbin JA, Sleeper LA, Orav EJ, et al. The pediatric cardiomyopathy registry and heart failure: key results from the first 15 years. Heart Fail Clin. 2010; 6(4): 401-13. doi: 10.1016/j.hfc.2010.05.002. PMID: 20869642, PMCID: PMC2946942.

3) Hong YM. Cardiomyopathies in children. Korean J Pediatr. 2013; 56(2): 52-9. doi: 10.3345/kjp.2013.56.2.52. PMID: 23482511, PMCID: PMC3589591.

4) Lipshultz SE, Sleeper LA, Towbin JA, Lowe AM, Orav EJ, Cox GF, et al. The incidence of pediatric cardiomyopathy in two regions of the United States. N Engl J Med. 2003; 348(17): 1647-55. doi: 10.1056/NEJMoa021715. PMID: 12711739.

5) Elmasry OA, Kamel TB, El-Feki NF. Pediatric cardiomyopathies over the last decade: a retrospective observational epidemiology study in Ain Shams University, Children's Hospital (Cairo, Egypt). J Egypt Public Health Assoc. 2011; 86(3-4): 63-7. doi: 10.1097/01.EPX.0000399140.68151.6a. PMID: 21844761.

6) Towbin JA, Bowles NE. The failing heart. Nature. 2002; 415(6868): 227-33. doi: 10.1038/415227a. PMID: 11805847.

7) Jefferies JL, Towbin JA. Dilated cardiomyopathy. Lancet. 2010; 375(9716): 752-62. doi: 10.1016/S01406736(09)62023-7. PMID: 20189027.

8) Moak JP, Kaski JP. Hypertrophic cardiomyopathy in children. Heart. 2012; 98(14): 1044-54. doi: 10.1136/heartjnl-2011-300531. PMID: 22591735.

9) Maron BJ. Hypertrophic cardiomyopathy: a systematic review. JAMA. 2002; 287(10): 1308-20. doi: 10.1001/jama.287.10.1308. PMID: 11886323.

10) Cooper LT, Baughman KL, Feldman AM, Frustaci A, Jessup M, Kuhl U, et al. The role of endomyocardial biopsy in the management of cardiovascular disease: a scientific statement from the American Heart Association, the American College of Cardiology, and the European Society of Cardiology. Endorsed by the Heart Failure Society of America and the Heart Failure Association of the European Society of Cardiology. J Am Coll Cardiol. 2007; 50(19): 1914-31. doi: 10.1016/j.jacc.2007.09.008. PMID: 17980265.

11) Semsarian C. Cardiac Genetics Diseases Council Writing Group. Guidelines for the diagnosis and management of hypertrophic cardiomyopathy. Heart Lung Circ. 2011; 20: 688-90. doi: 10.1016/j.hlc.2011.07.017. PMID: 22000299.

12) Oh JH, Hong YM, Choi JY, Kim SJ, Jung JW, Sohn S, et al. Idiopathic cardiomyopathies in Korean children. 9-Year Korean Multicenter Study. Circ J. 2011; 75(9): 2228-34. doi: 10.1253/circj.CJ-11-0051. PMID: 21747192.

13) Saadallah AA, Rashed MS. Newborn screening: experiences in the Middle East and North Africa. J Inherit Metab Dis. 2007; 30(4): 482-9. doi: 10.1007/s10545-007-0660-5. PMID: 17701444.

14) Towbin JA, Lowe AM, Colan SD, Sleeper LA, Orav EJ, Clunie S, et al. Incidence, causes, and outcomes of dilated cardiomyopathy in children. JAMA. 2006; 296(15): 1867-76. doi: 10.1001/jama.296.15.1867. PMID: 17047217.

15) Huang M, Zhang X, Chen S, Sun Y, Xiao Y, Sun J, et al. The effect of carvedilol treatment on chronic heart failure in pediatric patients with dilated cardiomyopathy: a prospective, randomized-controlled study. Pediatr Cardiol. 2013; 34(3): 680-5. doi: 10.1007/s00246-012-0527-x. PMID: 23108482.

16) Saucedo NA, Alarcón AV, Islas GE, De La Cruz LR. Evaluation of the effect of carvedilol in children with dilated cardiomyopathy. Arch Cardiol Mex. 2008; 78(1): 52-9. PMID: 18581713.

17) Harmon WG, Sleeper LA, Cuniberti L, Messere J, Colan SD, Orav EJ, et al. Treating children with idiopathic dilated cardiomyopathy (from the Pediatric Cardiomyopathy Registry). Am J Cardiol. 2009; 104(2): 281-6. doi: 10.1016/j.amjcard.2009.03.033. PMID: 19576361, PMCID: PMC2746101. 